\title{
EL ESTADO COMO ACTOR INTERNACIONAL: CRISIS Y CONSOLIDACIÓN DEL SISTEMA DE ESTADOS
}

\author{
Esther Barbé \\ Profesora de Relaciones Internacionales \\ Facultat de Ciències Politiques i de Sociologia \\ Universitat Autònoma de Barcelona
}

Resum

L'estat ocupa un lloc privilegiat en les tipologies d'actors internacionals. Els elements de l'estat (territori, població $i$ govern) son analitzats en aquest article en tant que criteris d'ordre politic. D'aquesta manera el concepte juridic de sobirania, que estableix la igualtat entre els estats, és substituit en aquest article pel concepte de capacitat. En consequiencia, l'article aborda no l'estat com a subjecte únic del Dret, sinó els estats com a pluralitat, amb capacitats diferenciades entre ells. En aquest sentit, una revisio comparativa -uen termes demogràfics, territorials, economics i militars, entre d'altres- d'alguns dels cent vuitanta un membres (en el moment actual) de Nacions Unides, es significativa de la jerarquia existent en el sistema d'estats. Una jerarquia elaborada a partir d'una base tangible (els recursos), d'una base intangible $i$ de la voluntat politica dels estats respectius. En l'article es revisen les grans definicions de la dita jerarquia: superpotències, grans potències, potències mundials, potencies mitjanes i potències regionals, entre d'altres.

\section{Resumen}

El estado ocupa un lugar privilegiado en las tipologias de actores internacionales. Los elementos del estado (territorio, poblacion y gobierno) son analizados en el artículo en tanto que criterios de orden politico. Asi, el concepto juridico de soberania, que establece la igualdad entre los estados, es sustituido en este articulo por el concepto de capacidad. En consecuencia, el artículo aborda no tanto el estado en tanto que sujeto único del Derecho sino los estados en tanto que pluralidad, con capacidades diferenciadas entre sí. En este sentido, una revisión comparativa -en términos demográficos, territoriales, económicos y militares, entre otros - de algunos de los ciento ochenta y un estados miembros (en el momento actual) de Naciones Unidas, es significativa de la jerarquia existente en el sistema de estados. Una jerarquía elaborada a partir de una base tangible (los recursos), cada vez más difusa, de una base intangible y de la voluntad politica de los respectivos estados. En el artículo se revisan las grandes definiciones de dicha jerarquía: superpotencias, grandes potencias, potencias mundiales, potencias medias y potencias regionales, entre otras. 
"Papers»: Revista de Sociologia

Abstract

The state occupies a privileged place in the typologies of International Actors. The elements of the state - territory, population and government-are analyzed in this article in terms of criterion of a political order. The legal concept of sovereignity which stablishes equality between states is substituted in this article by the concept of capacity. As a result this article deals with the state not so much as the sole subject of law but the state in terms of plurality with different capacities. In this sense a comparative revision - in terms of demography, territory, economics and the military, among others, of some of the one hundred and eighty-one member states in the present moment of the United Nations- reflects the existing hierarchy in the system of states.

Hierarchy is developped on a tangible basis (resources), which is more and more diffuse, an intangible basis and the political will of the respective states. In the article the broad definition of this hierarchy is summarised: Superpowers, Great Powers, World Powers, Medium Powers and Regional Powers, among others.

Cualquier estudio sobre actores internacionales comienza por una referencia al estado. En algunas ocasiones porque el estado es presentado como actor privilegiado o casi único, como en el caso de autores realistas clásicos (Morgenthau, 1948). Sin embargo, en otras ocasiones el analista dedica su obra a demostrar el papel cada vez más reducido de los estados (gobiernos nacionales) en el sistema internacional, tal y como podemos comprobar en el artículo de C. García (en este mismo volumen). De esta manera, en tanto que afirmación o en tanto que negación, el estado se convierte en referencia continuada en las relaciones internacionales. En esa misma lógica, crisis y consolidación del estado (como actor internacional) se manifiestan en paralelo, tal y como veremos a lo largo de este artículo.

La consolidación del estado como forma de organización política es una evidencia en términos numéricos. Sólo hay que recordar que la Organización de Naciones Unidas ha pasado de cincuenta y un miembros, firmantes originarios de la Carta de San Francisco en 1945, a ciento ochenta y uno ${ }^{1}$ en abrit de 1993. La fragmentación estatalizadora es un hecho cotidiano y, en ocasiones dramático, desde el final de la guerra fría.

La crisis del estado, por otra parte, está asociada al desafio que supone para los gobiernos nacionales otro tipo de actores. Así, el desarrollo de las organizaciones internacionales o la mundialización de la economía, acompañada del

1. Entre los estados de un cierto tamaño, no miembros de la Organización de Naciones Unidas por razones diferentes, se cuenran en la actualidad Suiza y las dos Coreas. Otros estados no miembros de la Organización se encuentran en su mayoria en el Pacífico y en el Caribe, tratándose de islas de pequeño tamaño y de descolonización tard́a. 
reforzamiento de las firmas multinacionales, son ejemplos en dicho sentido. Sin embargo, el papel del estado como actor internacional se ve desafiado por su propia proliferación, que supone la utilización de un mismo concepto para hablar de realidades absolutamente diferentes. Algunos autores consideran que, dada la diferencia existente entre los estados en términos de recursos, capacidades, posibilidades y capacidad para explotar y escoger entre todas esas posibilidades, es inapropiado liamar a todas esas unidades estados (Russett y Starr, 1989:61). Un simple ejemplo nos puede ayudar en términos comparativos: mientras los Estados Unidos de América tenían en 1990 una población de 250 millones de habitantes y un PNB de 5,5 billones de dólares, otro estado-miembro de Naciones Unidas (en este caso, San Cristobal y Nieves) tenía una población de 40.000 habitantes y un PNB de 133 millones de dólares ${ }^{2}$. De ahí que, en este artículo, una vez establecidos los elementos materiales de definición del estado, pasemos a hablar en plural: los estados frente al estado.

\section{SOBERANIA Y TERRITORIO}

La aparición del estado en su forma actual es relativamente reciente. En términos internacionales - creación de un sistema de estados-el Tratado de Westfalia (1648) supone el punto de partida de un nuevo mundo, en el que la anterior multiplicación de autoridades y de lealtades (príncipes, emperador, Papa) da paso al monopolio de la autoridad política sobre un territorio determinado. El principio central del Tratado de Westfalia era simple —el monarca impondrá su religión en su territorio- pero a la vez novedoso: el monarca sería soberano en su territorio. Desaparecía, de esta manera, la posibilidad de una autoridad externa o superior a la del monarca (emperador, Papa). Mientras en Europa se imponía el sistema de estados, en otras zonas del mundo como en India, en China o entre los árabes o los mongoles, persistía el sistema imperial.

Los elementos constitutivos del estado moderno son tres: territorio, población y gobierno, con autoridad para gobernar sobre los otros dos. Jurídicamente, el estado se diferencia de cualquier otra entidad territorial porque goza de un status legal único, goza de soberanía; lo que diferencia al estado de cualquier otro actor y lo que iguala a los estados entre sí. En términos juridicos, todos los estados son iguales, en tanto que todos son soberanos.

Hedley Bull (1977:8) indica que la soberanía incluye «la soberanía interna,

2. Los datos estadisticos, a no ser que se indique lo contrario, proceden del Anuario El País 1993, de L'etat du monde 1993 y del Informe sobre el Desarrollo Mundial 1992. 
que comporta la supremacía sobre cualquier otra autoridad existente entre la población o en el territorio" y la soberanía externa que significa "no supremacía, sino independencia de cualquier autoridad externan. Así, la soberanía externa se traduce en que ninguna autoridad puede decir al estado cómo actuar. Es decir, que no existe ningún superior jerárquico con autoridad legítima para decir al estado lo que tiene que hacer. Es independiente. Lo que algunos autores, como Raymond Aron (1962), traducen en el carácter legítimo del recurso a la fuerza armada por parte del estado y otros, desde el Derecho Internacional, apuntan que "soberanía no significa que el estado no esté sometido a reglas obligatorias que le son superiores; significa que no está sometido sin su consentimiento a ninguna autoridad ni organismo que le imponga una obligación" (Colard, 1991:87).

Ahora bien, la independencia objetiva del estado (declaración de independencia) alcanza todo su valor en el sistema internacional cuando es reconocida por los otros miembros del sistema. A veces ese reconocimiento puede tener efectos importantes sobre el propio sistema: la proclamación de independencia de Croacia y Eslovenia, en junio de 1991, se convierte en términos del sistema de estados europeos en un desafío de primer orden. En ese sentido, la postguerra fría nos ha traído ejemplos evidentes de cómo los estados independientes precisan del reconocimiento de la comunidad internacional. En especial, de aquellos estados o grupos de estados considerados vitales para la supervivencia del nuevo estado (vecinos poderosos, grandes potencias, etc.). De manera genérica se puede apuntar que, desde las grandes descolonizaciones de los años sesenta, el ingreso del estado recién nacido en Naciones Unidas es el corolario de su reconocimiento por la comunidad internacional: una cierta legitimación colectiva. El reconocimiento es el ejemplo de partida que nos muestra que toda consideración sobre el papel de un estado en el sistema internacional se ha de expresar en términos de relación (el poder del estado, como veremos más adelante, no se considera per se, sino en un marco de relaciones determinado entre dicho estado y los otros actores del sistema internacional).

En el terreno jurídico, la relación entre estados soberanos viene determinada en la actualidad por una serie de principios contenidos en la Carta de Naciones Unidas. Entre los cuales destacan tres grandes principios:

1. el principio de la igualdad entre los estados (artículo 2.1) queda reflejado en la composición de la Asamblea General de Naciones Unidas, donde se aplica la regla de "un estado, un voto»;

2. el principio de la no-intervención o de la no-injerencia (artículo 2.7) se puede interpretar como el corolario lógico de la soberanía;

3. el principio de no recurrir a la fuerza (artículo 2.4) tiene por objeto que un estado no recurra a la amenaza o al uso de la fuerza contra la inte- 
gridad territorial o la independencia política de otro estado y, con ello, la guerra se ve abolida, salvo en casos de legítima defensa (artículo 51).

La experiencia de cuarenta y ocho años de funcionamiento de Naciones Unidas nos sirve para saber que los principios han sido habitualmente vulnerados. Es más, en los últimos años se puede hablar de una corriente amplia de opinión que se plantea la revisión de algunos principios, como en el caso de la no-injerencia. En realidad, las mismas resoluciones del Consejo de Seguridad de Naciones Unidas, en casos como la asistencia a los kurdos del norte de Irak en 1991 o a la población de Somalia en 1992, están vulnerando dicho principio en nombre del derecho humanitario.

Entre las características apuntadas nos interesa retener el status legal del estado -su soberanía-, que le diferencia de cualquier otro actor internacional. Si bien hay que precisar que el estado tiene una segunda característica que le diferencia de los demás actores: la disposición de territorio terrestre, aéreo y marítimo, a no ser que se trate de un estado enclavado o sin salida al mar. Ningún otro actor dispone legalmente de un territorio. Hoy en día, todo el territorio del mundo (la Antártida en situación de impasse) está dividido entre estados. Así, el conjunto del planeta está dividido entre doscientas veinticinco unidades políticas ${ }^{3}$.

Los criterios jurídicos nos dan un punto de partida que, desde las relaciones internacionales, hemos de relativizar en base a la capacidad y a la habilidad de cada entidad soberana para ejercer un efectivo control sobre su territorio y para llevar a cabo sus objetivos (a nivel nacional y a nivel internacional). Así, más allá del criterio jurídico definidor de toda entidad estatal (soberanía), el enfoque funcional de las relaciones internacionales se centrará en la habilidad de cada entidad para movilizar recursos de cara a la consecución de objetivos determinados y en la capacidad de la misma para ejercer influencia sobre el comportamiento de otros actores del sistema internacional.

La soberanía es un concepto relarivo en términos de influencia internacional. ¿En qué medida es soberano un estado gravemente endeudado ${ }^{4}$ como Po-

3. A los ciento ochenta y un miembros de Naciones Unidas hay que sumar esrados que no son miembros de la Otganización, como Suiza y las dos Coreas, y territorios con estatutos jurídicos parciculares. El número de unidades es muy flucruante. Así, el Anuario El País 1993 que aparece con doscientas veinritrés unidades (siguiendo el censo realizado por Naciones Unidas) ya se ha visto superado por el reconocimieneo internacional, y el ingreso en la ONU, de Macedonia (abril 1993) y por la división de Checostovaquia en dos estados.

4. Defunición del Banco Mundial, según la cual se trata de un grupo de quince países que se considera han tenido graves dificultades para atender al servicio de la deuda extema. Se definen como los países en los que tres de los cuatro coeficientes clave, promediados a lo largo del período 1988-90, se encuentran por encima de un nivel crítico, a saber: relación entre la deuda y el PNB $(50 \%)$, relación entre la deuda y la exportación de bienes y de todos los servicios (275\%), relación entre el servicio de la deuda acumulado y la exportación (30\%) y relación entre los incereses devengados y la exporración (20\%) (Informe sobre el Desarrollo Mundial, 1992:xii). 
lonia, Ecuador o Costa de Marfil? ¿En qué medida ejerce su soberanía un país que no dispone de flota para controlar la pesca ilegal en sus aguas territoriales? ¿Cómo hacer frente al uso de satélites científicos o militares que investigan sobre territorios ajenos? ¿Cómo puede un estado altamente desarrollado, como Austria, evitar los efectos nocivos sobre su medio ambiente de un vecino altamente contaminante como la República Checa?

La capacidad -en términos de riqueza/pobreza, entre otros-será un criterio determinante en este artículo pero, tal y como mostraba el ejemplo checho-austriaco, el poder en las relaciones internacionales es un fenómeno cada vez más difuso y más difícil de determinar en el entramado global del sistema internacional.

\section{LOS ESTADOS FRENTE AL ESTADO}

Los actores internacionales, tal y como nos muestra C. García en este volumen, constituyen un mundo similar al reino animal. Son numerosos, variados por su naturaleza, cumplen funciones diversas, evolucionan, etc. Al igual que a los animales los clasificamos en clases y una vez establecidas las grandes clases (mamíferos o estados, reptiles u organizaciones internacionales, etc.) entramos en las subclases, los órdenes, las familias y las especies. Este símil sirve para indicar, antes de comenzar a hablar de los estados en el sistema internacional, que no existe una única clasificación a realizar. Al contrario, criterios diversos nos permitirán clasificar a esos ciento ochenta y un estados que forman la ONU en múltiples subclases (y a partir de ahí reclasificarlos nuevamente según criterios más restringidos). Algunos autores hablan de uclubs de estados": los ricos, los nuclearizados, los exportadores de combustible 5 , los militarizados, los estados-bomba, etc.

A modo de ilustración, y sin ánimo exhaustivo, podemos abordar la idea de la diferencia en el sistema de estados a partir de algunos criterios tangibles que, más adelante, nos van a servir de base para establecer el concepto de jerarquía entre los estados del sisterna.

La diferencia entre los estados (en su forma actual) viene, en primer lugar, dada por un elemento obvio: su edad. En la actual Asamblea General de $\mathrm{Na}$ ciones Unidas se sientan estados jóvenes (apenas una década de existencia y sin tradición previa como entidad política) junto a estados viejos (varios siglos

5. Los países exportadores de combustible, distinguidos como rales por el Banco Mundial, son aquellos países cuyas exportaciones y reexportaciones de petróleo y gas representaron como mínimo un $50 \%$ de sus exportaciones en el periodo de 1987-89. Entre estos países se encuentran Arabia Saudita, Emiratos Arabes Unidos, Libia, Nigeria o Venezuela (Informe sobte el Desarrollo Mundial, 1992:xi). 
de existencia y tradiciones asentadas como entidad política y/o culrural). Si bien es dificil abordar comparativamente el sistema de estados de principios de siglo (mucho más heterogéneo y complejo en términos jurídico-territoriales) y el actual, sí se puede apuntar la diferencia obvia que existe entre la cifra de cuatenta y dos estados existentes en el año 1900 y la presente pertenencia a la ONU de ciento ochenta y un miembros. La Asamblea General de la ONU es un ejemplo clato del actual sistema: el crecimiento entre 1950 y 1976 , de sesenta y cuatro a ciento cuarenta y siete miembros supone la entrada en la Organización de un mundo nuevo (afro-asiático), racial y culturalmente alejado del mundo ginebrino (europeo-americano), que era el marco de la Sociedad de Naciones en los años treinta de este siglo. Así, en 1934, de los cincuenta y ocho miembros de la Sociedad de Naciones tan sólo nueve eran afroasiáticos (tres africanos y seis asiáticos) frente a veintiocho europeos y a diecinueve americanos; mientras que en 1976, ochenta y dos miembros de la ONU eran afro-asiáticos (cuarenta y nueve africanos y treinta y tres asiáticos) frente a treinta y un europeos o a veintinueve americanos.

La descolonización masiva en la década de los sesenta significó la aparición de una multitud de estados que, en gran medida, se delimitaron a partir de fronteras administrativas de origen colonial y que carecían de recursos humanos adecuados (ingenieros, médicos, diplomáticos, etc.) para dirigir y gestionar el país. De manera genérica ${ }^{6}$, se puede apuntar que los jóvenes estados tienen enormes handicaps en comparación a los viejos estados. Handicaps en múltiples sentidos: pobreza, falta de infraestructuras, sociedades no consolidadas dentro de sus fronteras, irredentismos ligados a reafirmaciones nacionalistas, etc.

La fragilidad de dichos estados es obvia en Africa, donde la Organización de la Unidad Africana (creada en 1963) convirtió en un principio básico el respeto de las fronteras surgidas de la descolonización. Una vez más, la postguerra fría ha permitido la vulneración de principios asumidos. Así, tras décadas de lucha armada contra el ejército etiope, el referéndum de autodeterminación celebrado en Eritrea (abril 1993), con la supervisión de Naciones Unidas y favorable a la secesión de dicho territorio significa la apertura de una nueva era "legal" para los estados africanos. La fragmentación de estados "congelados" durante la guerra fría y la aparición de otros nuevos, con el consiguiente aumento de miembros en Naciones Unidas, es una tendencia evidente en los años noventa.

6. Nos referimos a tendencias genéricas. Algunos estados de «edad avanzada", radicados en América Latina, comparren las dificuliades de la mayor parte de estados jóvenes del Tercer Mundo, mientras que algunos estados recientes (caso de Bruncio de algunos países del Golfo) tienen, en cambio, una situación diferente debida al azar: la posesión de abundantes recursos energéticos en sus territorios. 
Tras la edad, otros dos elementos tangibles nos permiten apreciar la diversidad actual entre los estados: su territorio y su población. En términos territoriales, el planeta está dividido en doscientas veinticuatro unidades que van desde las dimensiones de Rusia (diecisiete millones de kilómetros cuadrados) hasta las dimensiones del Vaticano (un kilómetro cuadrado). Los extremos no son, sin embargo, elocuentes de un hecho evidente: el $50 \%$ del territorio mundial está bajo la soberanía de ocho estados (Rusia, Canadá, China, Estados Unidos, Brasil, Australia, India y Argentina) y cuarenta y ocho unidades (estados y territorios) tienen menos de mil kilómetros cuadrados (el municipio de Madrid tiene seiscientos kilómetros cuadrados). Entre estos últimos se encuentran estados miembros de Naciones Unidas, como Bahrein $\left(622 \mathrm{~km}^{2}\right)$, San Vicente y las Granadinas $\left(388 \mathrm{~km}^{2}\right)$ o Liechtenstein $\left(157 \mathrm{~km}^{2}\right)$, que tienen superficies equivalentes a lo que en España son algunos parques nacionales $\left(\right.$ los $750 \mathrm{~km}^{2}$ de Doñana o los $160 \mathrm{~km}^{2}$ de Ordesa-Monte Perdido).

El tamaño del territorio, sin embargo, no es un valor per se (Rusia o Gran Bretaña, con territorios muy diferentes, han jugado en diferentes momentos papeles de primeras potencias, respectivamente continental y marítima). La ubicación del territorio (control de estrechos estratégicos, país enclavado, condiciones climáticas, etc.) y su riqueza (combustibles, minerałes estratégicos, autosuficiencia alimentaria, agua, etc.) son cuestiones a considerar. En lo que respecta a la riqueza del territorio, no hace falta recordar la trascendencia polírica que ha tenido la concentración de los recursos energéticos en algunas regiones del mundo, destacando los países árabes con el $60 \%$ de las reservas mundiales de petróleo. Los recursos de agua dulce, por ejemplo, se han convertido en plena década de los noventa en un tema políticamente preocupante. Tanto es así que las disputas internacionales a causa del agua - por ejemplo, el agua del Nilo entre Egipto, Sudán y Etiopía o el agua del Tigris y el Eufrates entre Irak, Siria y Turquía - constituyen un tema destacado en la agenda internacional de finales del siglo XX.

La población, al igual que el territorio, está repartida de manera dispar a lo ancho del planeta. Los extremos en este caso corresponden a China, con una población en 1990 de 1.134 millones de habitantes, y al Vaticano con 1.000 habitantes. Seis esrados gozan de soberanía sobre el $50 \%$ de la población mundial (China, India, Estados Unidos, Indonesia, Brasil y Rusia), mientras que setenta y dos unidades (estados y territorios) tienen poblaciones de menos de un millón de habitantes. Entre estos últimos se cuentan miembros de Naciones Unidas como Gambia ( 875.000 habitantes), Islandia (258.000 habitantes) o Seychelles ( 68.000 habitantes). En otros términos, provincias o ciudades españolas tienen poblaciones equivalentes a las de un estado con escaño en la ONU: la población de Gambia es equivalente a la de 
la provincia de Zaragoza, la de Islandia a la del municipio de Hospitalet de Llobregat y la de Seychelles a la de la ciudad de Toledo.

Si el tamaño del territorio ha de contrastarse con otros muchos elementos cuantificables (riqueza natural, por ejemplo), lo mismo ocurre con la población; incluso más que en el caso anterior, ya que la "calidad" de la población, en términos demográfico-culturales, es un factor decisivo para el desarrollo de un estado. En este terreno (educación, cultura, salud) los indicadores a considerar son muy diversos: esperanza de vida, mortalidad infantil, analfabetismo, libros publicados, etc. Tan sólo recordar, a modo de ejemplo, y para abundar en la idea de la diferencia existente en el mundo contemporáneo entre unos y otros estados, que la tasa de mortalidad infantil en el año 1990 variaba, en los extremos, entre las 166 muertes por cada 1000 nacimientos de Malí y las 5 de Japón. La esperarza de vida oscilaba en el mismo año entre los 43 años de Guinea (idéntica para hombres y mujeres) y los 82 (para las mujeres) de Japón o Suiza. En lo que respecta a la tasa de analfabetismo, ésta va desde el $82 \%$ de Burkina Faso hasta una tasa habitual en los países de la OCDE inferior al 5\%.

\section{ESTADOS RICOS / ESTADOS POBRES}

Otros dos criterios, clásicos a la hora de abordar las diferencias tangibles entre los estados, son el tamaño económico y el tamaño militar de cada unidad política.

En lo que respecta al tamaño económico del estado, el PNB constituye un primer indicador a considerar. En 1990, el PNB de los Estados Unidos encabezaba el ranking mundial con 5,45 billones de dólares. En el extremo opuesto, el PNB del estado caribeño de Anguila apenas superaba los 8 millones de dólares. En el curso 1990 la Universitat Autònoma de Barcelona tenía un presupuesto de 18.200 millones de pesetas, más elevado durante ese año que el PNB de aproximadamente veintisiete unidades políticas del mundo (entre las que se encuentran Andorra, Kiribati, Maldivas o miembros de $\mathrm{Na}$ ciones Unidas, como San Cristóbal y Nieves).

Como siempre, más allá de los extremos, lo elocuente es observar la concentración del producto a nivel mundial. En efecto, tres estados (Estados Unidos, Japón y Alemania) acumulan más del $50 \%$ de la producción mundial (España ocupaba en 1990 el octavo puesto con un 2,1539\% del total mundial). Sólo dieciocho unidades políticas tenían en 1990 productos nacionales superiores al $1 \%$ del producto mundial, mientras que las ciento veintiséis unidades menos productivas del mundo sumaban en total un $1 \%$ de la producción mundial. Entre estas ciento veintiséis unidades encontramos a países como Panamá, Senegal, Estonia, Jordania, etc. 
EI PNB per capita es el siguiente indicador a considerar. Entre otras cosas, porque ha servido de base al Banco Mundial para diseñar una clasificación aplicable a todos los estados del mundo. El PNB per capita nos muestra que en las últimas décadas las diferencias van en aumento entre el sector privilegiado de estados (cada vez más reducido) y los demás. Según el Informe sobre el Desarrollo Humano 1992, en 1969 el $20 \%$ de la población del mundo que vivía en las naciones con mayor ingreso per cápita estaba treinta veces mejor que el $20 \%$ que ocupaba el rango inferior, mientras que en 1989 esa disparidad se había casi doblado, de manera que los más ricos eran sesenta veces más ricos y los más pobres seguían igual de pobres (PNUD 1992:2). Lo que de manera gráfica queda recogido en la diferencia abismal que separa a un habitante de Liechtenstein con 34.000 dólares de renta anual (el primer PNB per capita del mundo en 1990) de uno de Mozambique con 80 dólares (el último).

Como decíamos, el Banco Mundial clasifica los estados en tres grupos, según su nivel de PNB per capita: países de ingreso bajo, de ingreso mediano (subdividido en países de ingreso mediano bajo y países de ingreso mediano alto) o de ingreso alto.

En 1990, el grupo de los países de ingreso alto (un PNB per capita de 7.620 dólares o más) sumaba un total de cincuenta y cinco unidades políticas (junto a estados hay que incluir territorios como Hong Kong, Islas Vírgenes, Macao, etc.). Entre las cuales se cuenta España, con 10.920 dólares.

El grupo de países de ingreso mediano (con un PNB per capita entre 610 y 7.620 dólares) sumaba un total de ciento trece países, de los cuales ochenta y uno estaban por debajo de la línea de los 2.465 dólares (es decir, de ingreso mediano bajo). En este segundo grupo se incluyen países como Grecia, Portugal, Corea del Sur, Irak o Rusia.

Finalmente, el grupo de los países de ingreso bajo (inferior a 610 dolares) sumaba en 1990 un total de cincuenta y cuatro estados. Calificados de países pobres (World Resources, 1992-93:29), éstos se concentran en el África subsahariana y en el sur de Asia y, de manera más puntual, hay que mencionar Haití y países del sudeste asiático (Vietnam, Laos o Camboya).

El aumento espectacular de las diferencias entre países pobres y países ricos en las últimas décadas, asf como el deterioro social generalizado en el Tercer Mundo y en algunas capas sociales de los países ricos ha llevado al PNUD (Programa de Naciones Unidas para el Desarrollo) a buscar indicadores que permitan captar - mejor que cifras macroeconómicas de carácter global para un estado- el nivel de desarrollo humano. De ahí, la definicion del índice de desarrollo humano, ampliamente utilizado en la actualidad por el sistema de Naciones Unidas, y formado a partir de indicadores básicos sobre longe- 
vidad, educación y niveles de vida decentes de la población en cada estado. Se considera más explicativo que el PNB per capita (sobre todo en el caso de estados con enormes desigualdades sociales), ya que refleja la forma en que el crecimiento económico se traduce en bienestar humano. Es indicativo, en este sentido, que en el Informe sobre el Desarrollo Humano del año 1991, hubiera veintiséts estados cuya clasificación según el IDH estaba, como mínimo, veinte lugares por debajo de su clasificación según ingreso per capita (por ejemplo, los Emiratos Árabes Unidos con un puesto diecisiete en términos de ingreso en el año 90 ocupaba el puesto cincuenta y seis en lo que respecta al IDH del Informe 1991). Si nos atenemos a la clasificación elaborada por el PNUD en 1991, la primera sociedad del mundo por su nivel de desarrollo humano es la de Japón (seguida por Canadá, Islandia, Suecia y Suiza) y la última, la de Sierra Leona, precedida en los catorce puestos anteriores siempre por países africanos.

\section{ESTADOS ARMADOS}

El tamaño militar del estado constituye un criterio clásico a la hora de establecer jerarquías en el sistema internacional. Un indicador relevante a la hora de abordar el grado de militarización de un estado es la relación entre el presupuesto militar del estado y su PNB. Con ese criterio, a lo largo de la última década, los países más militarizados del mundo se sitúan en la zona de Oriente Medio. Irak, que en el año 1990 dedicaba el $20 \%$ de su PNB a gastos militares, había llegado en 1984 a dedicar prácticamente el 30\% de su PNB a dichos gastos. Israel, que es otro caso de estado militarizado, ha pasado de un $23,5 \%$ en 1981 a un $8,4 \%$ en 1990 . En Estados Unidos el porcentaje oscila entre el $5,6 \%$ de 1990 y el 6,7\% de 1986 , mientras que los estados europeos de la OTAN se mueven, entre 1981 y 1990 , con tendencia a la baja en los últimos años, entre los mínimos de Luxemburgo (alrededor del 1\%) y los máximos de Grecia (que superó el $7 \%$ a mediados de los ochenta). España, por su parte, se mueve entre un máximo del $2,4 \%$ y un mínimo (en 1990) del $1,8 \%$. Desgraciadamente, en muchos casos los países pobres son los que ocupan puestos destacados por su gasto militar. La situación de guerra civil o de regímenes dictatoriales en países del Tercer Mundo alimenta buena parte de la industria armamentista. Angola ha líegado a consumir a mediados de los años ochenta el 30\% de su PNB en gastos militares; Etiopía gastó en 1989 el 13,5\%; Cuba llegó hasta el 11,3\% en 1988; Libia, al 15\% en 1985; Nicaragua, al $34,2 \%$ en 1987; etc. La lista de los doce mayores importadores de armas, entre 1985 y 1989, reune a un grupo hererogeneo de países (India, Irak, Japón, Arabia Saudí, Siria, Egipto, Checoslovaquia, Corea del Norte, España, 
Polonia, Turquía y Angola) que, en algunos casos (España y Checoslovaquia, por ejemplo), son a su vez exportadores ${ }^{7}$.

En términos globales, la "capacidad de matar" está distribuida de manera extremadamente desigual; si bien no se corresponden necesariamente las regiones de mayor potencialidad armada (posesión de armamento nuclear y convencional) con las regiones de mayor conflictividad armada. Así, en 1989 (bajo la lógica aún del sistema bipolar) el poder para matar (killing power) de las dos superpotencias justificaba la propia lógica del sistema: la letalidad de las superpotencias (sumadas armas nucleares, quimicas y convencionales) era, respectivamente, de un $41 \%$ para los Estados Unidos y de un $57 \%$ para la Unión Soviética, del total mundial. El resto del mundo gozaba de un $2 \%$ de letalidad. Lógicamente, dejando de lado el armamento nuclear de carácter disuasorio y entrando en el terreno del armamento convencional, de uso militar cotidiano en las treinta y una guerras abiertas en el año 1989, las cifras variaban ostensiblemente: la Unión Soviética con el $24 \%$ de potencial armado, sumado al $6 \%$ de los otros países del Pacto de Varsovia, y los Estados Unidos con el $18 \%$, sumado al $11 \%$ de los otros países de la OTAN, seguían jugando un papel destacado. Pero, junto a estas cifras, el $12 \%$ de Oriente Medio o el $8 \%$ de China nos muestran el carácter multipolar del sistema internacional en términos de potencial de destrucción militar ${ }^{8}$. No hay que olvidar que en el momento del inicio de la Guerra del Golfo (1991), el ejercito irakí estaba considerado como el cuarto ejército del mundo tras el estadounidense, el soviético y el chino.

¿Cómo vamos a establecer la jerarquía entre los estados del actual sistema internacional? Hemos mencionado recursos diversos (territorio, población, riqueza, fuerzas armadas) que todo estado poderoso ha poseido y posee en cantidades destacadas. Muchas veces es, en base a dichos recursos, que se juzga el poder de un estado en el sistema internacional. Así, se asume que los estados grandes siempre son capaces de obligar a los pequeños a actuar de acuerdo con los intereses de los primeros. De esta manera, se está abordando el poder en el sentido hobbesiano, en términos de cantidad o de propiedad: el poder como posesión. Sin embargo Locke, Max Weber $o$, más recientemente, los analistas de las relaciones internacionales distinguen entre el poder como recurso (la base del poder) y el poder como relación (la influencia de un estado sobre los demás). Esto es, el poder sólo se puede entender como una relación entre estados y la estimación del poder debe realizarse en las precisas circunstancias en las que la interacción tiene lugar (Hocking y Smith, 1990:195). Sobre el papel, los

7. Entre 1987 y 1991 España era el país número doce del mundo por su nivel de venta de armas al mundo en desarrollo y el número trece en lo que respecta a su nivel de ventas al mundo industrializado (SIPRI, 1992:272).

8. Las cifras están extraidas de Kidron, M. y Smith, D. (1991) y de SIPRI (1991). 
recursos de los Estados Unidos en los años sesenta y setenta frente al enemigo vietnamita eran muy superiores. Su poder potencial, sin embargo, no se tradujo en ejercicio del poder (obtención de los objetivos perseguidos) y los Estados Unidos acabaron perdiendo una guerra en la que, en términos potenciales, tenían que conseguir la victoria. Lo mismo se puede apuntar en relación con Francia y la guerra de independencia de Argelia o con la Unión Soviética en Afganistán.

\section{LA INTANGIBILIDAD DEL PODER}

La evaluación del poder en términos internacionales es un proceso complejo que dificilmente puede ser cuantificado. Algunos investigadores se han dedicado, con escaso éxito, a establecer correlaciones entre diversos indicadores y a construir índices de poder con la intención de identificar las potencias, y de jerarquizar así los estados del sistema internacional?.

El proceso de transformar unos recursos cuantificables en el ejercicio de una cierta influencia en el sistema internacional (obligar a otro estado, de modo coercitivo o no, a que actue según nuestra voluntad) supone una movilización en la que recursos intangibles van a estar presentes. En muchas ocasiones se trata de la otra cara de los recursos tangibles, conocidos y cuantificables (territorio, fuerzas armadas, población, riqueza económica). Por ejemplo, ¿cómo podemos cuantificar la moral de una población?, ¿cómo podemos valorar la capacidad de liderazgo de los oficiales de un ejército?, ¿cómo podemos prever la eficacia de un cuerpo diplomático?, etc. Así, junto a los recursos que dotan al estado de una capacidad determinada (tener poder), éste debe gozar también de las estructuras políticas, sociales y económicas que permitan al gobierno movilizar dichos recursos nacionales y convertirlos en instrumentos de política exterior, con el objetivo de ejercer influencia internacional (ejercer poder).

Sin ánimo de exhaustividad se pueden mencionar algunos recursos intangibles característicos. La cohesión de la población es un factor importante. Así, en aquellos países en los que un grupo (tribu, grupo religioso, etc.) se sienta ajeno al estado, éste tendrá una fuente de debilidad. El levantamiento de chiitas y de kurdos contra el gobierno de Sadam Hussein durante la guerra de 1991 es un ejemplo en este sentido. El nivel de compromiso individual con la persistencia del estado es otro aspecto importante que explica, entre otras cosas, que algunos estados puedan persistir en un medio hostil. El caso de Is149).

9. Véase el análisis sobre dichos proyectos de investigación en Russett y Starr (1989: 138- 
rael en el seno del mundo árabe es un buen ejemplo. Algunos factores tan intangibles, como el prestigio, están ligados a la actuación de determinadas personalidades políticas: el peso de la Suecia de Olof Palme o de la Tanzania de Nyerere en los organismos internacionales. Elementos tan intangibles como la moral nacional pueden ayudar a entender los fracasos de Francia en Argelia y de Estados Unidos en Vietnam.

Algunos de los recursos intangibles apuntados (capacidad de liderazgo, moral nacional, eficacia administrativa, etc.) serán decisivos en el momento en que el gobierno se decida a traducir su capacidad nacional en una determinada influencia internacional. De modo particular hay que hacer mención concreta de un elemento imprescindible en el proceso de movilización de dicha capacidad: la voluntad política. Así, el proceso de ajustar determinados recursos (fuerzas armadas, medios de transporte, riqueza económica, cuerpo diplomático, etc.) a la persecución de ciertos objetivos (expansión territorial, funciones pacificadoras, responsabilidades organizativas internacionales, etc.) estará determinado por la voluntad de asumir funciones de dimensión internacional: colonización, "misión civilizadora», organización del mantenimiento de la paz, mediación internacional, etc. La voluntad, o la falta de voluntad, para asumir dichas funciones es básica a la hora de valorar la influencia que va a ejercer un estado. En este entido, ya es clásico mencionar que la influencia ejercida por los Estados Unidos en el sistema internacional entre principios de siglo y la Segunda Guerra Mundial es muy inferior a sus capacidades (recursos tangibles). Justamente por la falta de voluntad política (determinada por la falta de apoyo popular) que lleva a ese país a persistir en su tradicional política de aislamiento a pesar de los cambios ocurridos a nivel mundial (entre otros, la importancia cada vez mayor de los Estados Unidos en términos económicos, técnicos y militares). La no pertenencia de ese país a la Sociedad de Naciones, a pesar de que fue un proyecto concebido por el presidente Woodrow Wilson, es el mejor ejemplo en este sentido. Ejemplos más recientes, como el comportamiento de los países de Europa Occidental en las guerras de la ex-Yugosiavia, hacen reflexionar sobre la importancia de la voluntad política en la vida internacional, junto a la posesión de capacidades suficientes $y$ de instrumentos apropiados para alcanzar los objetivos perseguidos.

En el proceso de movilizar recursos para transformarlos en influencia, hay que tener en cuenta también la dificultad que supone en muchos casos el disponer de recursos apropiados para una situación dada. Es importante entender en qué medida los diferentes tipos de recursos y los instrumentos de influencia (diplomacia, disuasión militar, presión económica, etc.) pueden ser sustituidos los unos por los otros. En términos de inversión, por ejemplo, la riqueza puede ser utilizada para formar ejércitos modernos, una población culta y con amplios conocimientos (investigación y educación) o una población 
más sana. A su vez, los instrumentos militares pueden servir para adquirir más riqueza. Casi todas las bases de poder tienen cierta capacidad para transformarse en otras, pero el "tipo de cambio» varía mucho. En el terreno de los recursos intangibles es donde la dificultad es a veces más patente. ¿Cómo crear afecto entre una población predispuesta contra nosotros? El caso del rechazo entre la población somalí de las tropas multinacionales que desembarcaron en el país en diciembre de 1992, bajo bandera de Naciones Unidas, es un claro ejemplo en dicho sentido.

El problema de sustituir una base de influencia por otra crea dificultades para el analista de las relaciones internacionales que valora un tipo de recurso por encima de los demás; así los realistas con los recursos militares o los marxistas con los recursos económicos. En la década de los setenta, ligados a los cambios internacionales del momento, comienzan a aparecer múltiples trabajos que plantean la dificultad cada vez mayor para fundir un tecurso y transformarlo en otro. Así, la fuerza nuclear se muestra inútil a la hora de estabilizar un tipo de cambio o de disuadir a la OPEP de que aumente el precio del petróleo. La tendencia a dar cada vez mayor importancia al poder soft frente al poder hard (por ejemplo, sistemas de información frente a material militar) y a multiplicar los espacios de cooperación (las instituciones y los ámbitos multilaterales ganan en importancia) relega al pasado el discurso tradicional de los recursos militares como determinantes últimos de los poderosos en el sistema internacional: el poder es menos coercitivo (en términos militares).

En realidad, los años noventa son mucho más complejos que la Europa del Congreso de Viena a la hora de definir el poder de un estado, empezando por los propios riesgos para su seguridad nacional. El crecimiento del número de estados ha ido asociado con la vulnerabilidad de los mismos. Maquiavelo o Hobbes ya pensaban en términos de seguridad nacional, pero ¿qué inclúan ahf dentro? Basicamente integridad territorial. Hoy en día, el poder y también la soberanía del estado se ven afectados por fenómenos económico-ecológicos, como la deuda externa o la disminución de la capa de ozono. El poder en los años noventa es un poder difuso. Difusión que ha sido propiciada por la interdependencia económica, los actores transnacionales, el nacionalismo en los estados débiles, la extensión de la tecnología y los cambios en el escenario político (Nye, 1991:182).

\section{LAS REGLAS DEL JUEGO}

Hay que hacer una última distinción entre el poder como suma de recursos que los estados poseen (ya analizado) y la estructura externa que condicionan dicho poder. Lo que algunos autores definen como poder estructural: la ha- 
bilidad para determinar las reglas del juego en la política internacional. En otras palabras, el poder como relación. Así, el poder de un estado es tal en la medida en que éste es capaz de ejercer su poder sobre otros estados y en la medida en que es capaz de establecer las reglas de juego. En este sentido, es importante conocer con exactitud el contexto en el que se va a desarrollar la actividad del estado, ya que los recursos y los objetivos de un estado adquieren sentido sólo cuando se ponen en relación con los recursos y los objetivos de otros estados. Por ejemplo, la India se ha convertido en los años ochenta (entre 1985 y 1989) en el primer comprador de armas del mundo (por encima incluso de Irak). Si el objetivo de la India era sentirse segura frente a sus vecinos, es obvio que lo ha conseguido en el caso de Pakistán pero ni mucho menos en el caso de China. A pesar de los esfuerzos indios - la India llegó a gastar hasta el 7\% de su PNB en defensa, en un país donde el PNB per capita es de 350 dólares-, la estructura de poder militar en Asia es tal que China predetermina el contexto militar en el que se mueven los países del sur y del sudeste asiático.

Los límites que la estructura de poder impone a la actuación de los estados, bien sea a nivel mundial o a nivel regional, han sido ampliamente destacados por los autores neo-realistas (Waltz, 1979). Sin embargo, una de las grandes críticas que se hace a los autores que analizan el sistema internacional a partir exclusivamente de la estructura de poder — determinada durante varias décadas por la relación bipolar Estados Unidos-Unión Soviética - es que son incapaces de captar factores de cambio, tales como las transformaciones internas en dichos estados o bien el proceso de difusión que ha sufrido el poder en las relaciones internacionales (en muchas ocasiones a espaldas de los estados que supuestamente definen la estructura de poder a nivel internacional). El sistema internacional de la post-guerra fría es, sin lugar a dudas, un sistema en el que tendríamos grandes dificultades para establecer la estructura de poder (caso de existir una sola). La mención a la bipolaridad Estados Unidos-Unión Soviética como estructuradora del poder a nivel internacional nos permite entrar en el último punto de interés de toda jerarquización entre los estados del sistema internacional. ¿Cuáles entre esos estados juegan el papel de potencia en el sistema? ¿Podemos hablar de un solo tipo de potencias? ¿Existen varios tipos de potencias internacionales?

¿Qué estado o qué estados pueden ser definidos como potencias del actual sistema internacional? Es decir, qué estados establecen reglas de juego y qué estados disponen de recursos y son capaces de movilizarios para defender dichas reglas. Esta reflexión es un "lugar común" en los últimos años. Después de varias décadas de asumir, de manera simplista, la existencia de dos potencias que estructuraban un sistema bipolar, el hundimiento del bloque soviético planteó múltiples preguntas. Posiblemente, el acontecimiento que mejor marcó esa reflexión sobre el sentido y la naturaleza de una potencia de finales del 
siglo xx fue la Guerra del Golfo. Esa reflexión vino a ser el colofón de un debate importante entre los analistas de las relaciones internacionales sobre el declive de los Estados Unidos como potencia internacional (Kennedy, 1987).

En efecto, la Guerra del Golfo reune una serie de características de manual para hacernos comprender en todas sus dimensiones la capacidad de un estado para ejercer una influencia tal que lo convierta en potencia dentro del sistema. Es decir, que tenga en sus manos grandes posibilidades para establecer las reglas del juego. El análisis del comportamiento del gobierno estadounidense y los resultados del mismo nos permiten hablar de una política de potencia. ¿Cuáles son los elementos que la conforman? Los analistas dedicaron miles de páginas a abordar el tema ${ }^{10}$. Aquí, de manera muy sintética, podemos hacer una consideración ligada a los elementos establecidos en este mismo artículo.

El comportamiento de los Essados Unidos empieza por definirse como comportamiento de potencia. La voluntad política del gobierno queda recogida en el discurso del presidente Bush sobre los Estados Unidos como defensores del Derecho Internacional ( $\mathrm{y}$ más allá como creadores de un Nuevo Orden Mundial). El objetivo perseguido (la defensa del Derecho) tiene una traducción concreta en la liberación del territorio kuwaití, expulsando al ejército irakí.

¿Cuáles son los recursos aplicados a dicho objetivo? Como hemos visto, los recursos pueden ser muy diversos. Sin embargo, en este caso, la posesión de un aparato militar apropiado para las dimensiones de la operación y la posibilidad de costear económicamente la operación son decisivos. Las dudas sobre la capacidad de los Estados Unidos para imponer sus reglas del juego -utilización de la fuerza contra el ocupante irakí- estaban directamente relacionadas con sus dificultades económicas. Sin embargo, en este caso se produce un ejemplo clásico de fusión del poder, ya que los Estados Unidos utilizan sus recursos políticos para conseguir que el coste de la operación vaya a cargo de sus aliados (Japón y Alemania). En este caso, recursos intangibles como la existencia de consenso entre la sociedad americana en favor de la acción militar o la capacidad de liderazgo del presidente Bush entre la opinión pública de su país, son elementos que refuerzan el papel de potencia asumido por los Estados Unidos. Papel que se ve legitimado a través del Consejo de Seguridad de Naciones, que legaliza el recurso a la fuerza dejando la aplicación del mismo en manos de "los estados que cooperan con el gobierno de Kuwait» (Resolución 678). En términos de recursos intangibles, la legitimación internacional de la política de los Estados Unidos es un facror importantísimo en tanto que confirma una vez más la capacidad de dicho país para obligar (con-

10. Por su capacidad y sintesis y por su claridad, se recomienda la lectura de un articulo periodístico de William P'faff (1990). 
vencer, inspirar, etc.) a otros estados a actuar de acuerdo con la política marcada por el primero.

Cuando la capacidad de un estado - como en el caso de Estados Unidos y de la Guerra del Golfo- para imponer su política tiene consecuencias mundiales, nos estamos moviendo a una dimensión global del sistema internacional. El mismo fenómeno se puede producir a un nivel geográfico inferior (regional) o bien, en un área determinada de las relaciones internacionales (issue area), como las finanzas o el comercio.

A continuación vamos a establecer una tipología de potencias internacionales (sin ánimo de exhaustividad) y teniendo en cuenta que fuera de la misma quedan la mayor parte de estados del actual sistema internacional. Comenzábamos este artículo mencionando la diferencia "que salta a la vista" en términos numéricos entre los Estados Unidos de América y el estado de San Cristóbal y Nieves. Ambos nos sirven para definir una escalera que va desde los peldaños más elevados (la superpotencia) hasta el peldaño más bajo del actual sistema de estados (el fenómeno de los micro-estados). Estos últimos, defendidos por la lógica de la soberanía como principio universal e instrumentalizados por las mafias internacionales, pueden acabar legalizando aquello que las películas de James Bond nos mostraban como política-ficción: la aparición de alguna que otra "Spectra" con representación en la Asamblea General de Naciones Unidas. El asunto de los micro-estados ha sido tema de preocupación en la ONU: ¿cuál es la base mínima para gozar de la condición de miernbro de Naciones Unidas? San Cristóbal y Nieves tiene 40.000 habitantes. ¿Es una cuestión de población? ¿Es una cuestión de territorio? Y algunos se pueden preguntar, ¿por qué no una cuesrión de PNB?, abriéndose así una puerta que desde el Tercer Mundo se presiente peligrosa.

\section{SUPERPOTENCIAS, GRANDES POTENCIAS Y POTENCLAS MEDIAS}

Volviendo a la tipología de las potencias en el actual sistema internacional, hay que precisar, de entrada, que ésta se encuentra a caballo entre un sistema bipolar (desaparecido) y un sistema en formación (sin estructura de poder precisa). De ahi, por tanto, que sea preciso hacer referencia a algunos elementos del desaparecido sistema bipolar por lo que tienen de continuidad y de cambio en el actual sistema.

El sistema bipolar, y su progresiva transformación desde 1945 hasta 1989 , introducirá novedades en lo que respecta a la tradicional estructura de poder del sistema de estados europeos de los siglos XVIII y XIX, donde el concepto de gran potencia servía para definir a aquellos estados con recursos (más o menos equivalentes) de todo tipo (económico, polf́tico y militar) que creaban las re- 
glas del juego. El equilibrio del poder entre las grandes potencias (entre cinco y siete) constituía el mecanismo estabilizador del sistema. Pues bien, como es sabido, el sistema internacional surgido de la Segunda Guerra Mundial rompe con esa lógica previa. Además, la transformación progresiva del sistema (evidente a partir de los años setenta) hará cada vez más compleja la tipología de las potencias en ese sistema que nace en el 45 , se hunde en el 89 y nosotros revisamos en el 93. En este artículo vamos a simplificar hablando de la existencia de tres grandes tipos de porencias desde el final de la Segunda Guerra Mundial: Las superpotencias, las grandes potencias y las potencias medias.

Las superpotencias juegan en el sistema bipolar un papel equivalente al de las grandes potencias de la Europa del Congreso de Viena. Son las encargadas de establecer el equilibrio en el conjunto del sistema, no a través de la negociación diplomática (si bien ésta se utiliza desde los años sesenta), sino a través de la disuasión nuclear mutua, que constiruye el recurso para generar bloques ideológico-militares liderados por las dos superpotencias (Estados Unidos y Unión Soviética), pero que al mismo tiempo supone per se un factor de parálisis en tanto que lastra la capacidad de actuación (peligro de aniquilación mundial) y la economía de los dos Grandes. El alcance mundial de las superpotencias viene determinado, por tanto, por su poder de coerción (su capacidad militar y nuclear) que define la lógica bipolar. En esta definición se observa que la lógica bipolar domina el sistema en su dimensión político-militar y que, por tanto, hemos de hablar de dos superpotencias justamente en ese terreno. La desaparición del sistema bipolar no significa la desaparición de dichos recursos (militar-nucleares), sino la desaparición de las reglas del juego en dicho terreno; lo que genera incertidumbre en el actual sistema en transformación. La desaparición de la Unión Soviética ha supuesto una revisión del concepro de superpotencia, evidente de cara al futuro, pero también de manera retrospectiva a causa de la ausencia material de la Unión Soviética en la definición de las reglas del juego en el ámbito económico internacional. De ahí que el concepto de potencia hegemónica, en relación a la capacidad estadounidense de marcar las reglas del juego en el terreno económico (en solitario) y en el terreno político-militar (junto a la Unión Soviética), sea aplicado en ocasiones a los Estados Unidos como facror diferenciador respecto a la Unión Soviética durante la guerra fría. En buena medida, como veremos a continuación, la erosión respectivamente del papel de liderazgo ideológico de las dos superpotencias y del liderazgo económico de los Estados Unidos a partir de la década de los sesenta supondrá el reforzamiento de lo que aquí denominamos como grandes potencias (en el sistema bipolar y en la actualidad).

El concepto de gran potencia es aplicado a un grupo de países que cumplen funciones diferentes en el sistema. Sin duda, ese grupo de países está formado (desde la perspectiva de 1993) por Francia, Gran Bretaña, China, Japón y la 
República Federał de Alemania. Los criterios para destacar a este grupo de países son variados y entran en los ámbitos diversos de las relaciones internacionales (económico, político y militar). Como miembros permanentes del Consejo de Seguridad y del Club Nuclear, China, Francia y Gran Bretaña desempeñan un papel jerárquicamente superior en el sistema internacional que auna historia, prestigio y voluntad política. El papel de China, respecto a la Unión Soviética, y el de Francia, respecto a los Estados Unidos, como erosionadoras del liderazgo político-militar de las superpotencias es un factor destacable. Japón y la República Federal de Alemania, por su parte, consiguen gracias a sus recursos finacieros y comerciales convertirse, a su vez, en erosionadoras del papel hegemónico de los Estados Unidos en el ámbito económico internacional. Sin embargo, ninguno de los países mencionados se puede considerar tan completo como los Estados Unidos en lo que respecta a sus recursos (tangibles e intangibles) y a su voluntad política de ejercer como potencia con responsabilidades mundiales. Joseph Nye elabora un listado de recursos ${ }^{11}$ y somete a examen a los Estados Unidos, a la Unión Soviética, a Japón, a China y a Europa (en referencia a la Comunidad Europea), para flegar a a la conclusión de que en 1990 el único estado del mundo fuerte en todos ellos seguían siendo los Estados Unidos (Nye, 1991:174).

Finalmente, el concepto de potencia media es posiblemente el más abarcador, en tanto que recoge un grupo importante de países que destacan bien en un ámbito muy particular de la vida internacional o bien a un nivel regional. Este es un concepto muy utilizado en los últimos años en referencia a los países de tamaño medio (caso de México o Brasil en América Latina o de España e Italia en Europa), con una diplomacia activa en ciertas áreas (caso de la política iberoamericana de España o del papel de México y Venezuela en la pacificación de América Central), aunándose así recursos y voluntad política en la definición. En esta misma línea se puede destacar el papel de países de reducidos recursos (por su tamaño territorial, demográfico o económico), muy diferentes entre sí, pero que juegan un papel destacado en la diplomacia multilateral; es el caso de algunos pequeños países europeos: la Austria neutral de la guerra fráa, como espacio negociador, es un buen ejemplo. En este caso, el prestigio del país ligado a su voluntad «internacionalista» es el elemento destacable. Otro tipo de países que conforman la idea de potencia media son aquellos que por su peso (demográfico, económico, militar, etc.) en un marco regional juegan el papel de potencia en esa dimensión geográfica, estableciendo las reglas del juego en la misma. En este caso, la complejidad de algunas regiones hace que la asunción del papel de potencia constituya un tema per-

11. El listado de recursos estudiados es el siguiente: recursos tangibles (recursos básicos, militarización, economia, cienciá y tecnologia) y recursos intangibles (cohesión nacional, cultura universalista $e$ instituciones internacionales). 
manente de conflicto (India y Pakistán en Asia del Sur, Irak y Siria en el Próximo Oriente, Marruecos y Argelia en el Magreb, etc.). En este último caso, la bipolaridad solía ejercer un papel regulador que actualmente ha desaparecido; si bien, en otros casos, la bipolaridad suponía la persistencia por definición de un conflicto regional transformado en un conflicto bipolar (el enfrentamiento árabe-israelí).

\section{A MODO DE CONCLUSION}

En este artículo se ha intentado revisar, de manera general, un tema tan amplio como el papel del estado en el actual sistema internacional. La intención del mismo era destacar el carácter relativo de dicho actor internacional. Es decir, establecer que bajo un mismo concepto y unos mismos criterios jurídicos, el mundo de hoy en día observa realidades absoluramente diferentes, que van desde las superpotencias de la época de la guerra fría, capaces de aniquilar varias veces a la población mundial, hasta los paises africanos dependientes de la beneficencia, donde el gobierno es incapaz de ofrecer a su población los mínimos elementos de la supervivencia cotidiana.

Diferencia, por otra parte, que en términos económicos se ha ido agravando de manera espectacular en las últimas décadas. De ahí que la vulnerabilidad de muchos estados - población enferma y falta de recursos tan básicos como el agua para sobrevivir- relativice la continuación de esa forma de organización política. Lo que, junto a la actividad de algunos actores transnacionales (en especial firmas multinacionales) que burlan la soberanía de los estados y a la dimensión supranacional de los problemas medioambientales, podría llevarnos a creer en la crisis del estado.

Sin embargo, como se apunta en este mismo artículo, el crecimiento espectacular del número de miembros en Naciones Unidas (ciento ochenta y uno en abril de 1993) nos indica que dicha forma de organización política tiene un enraizamiento tal que se ha convertido en el motivo de múltiples guerras. El creciente nivel de conflictividad en el planeta - ligado a la desaparición de la bipolaridad y, con ella, de la Unión Soviética- está asociado a la voluntad estatalizadora de muchos pueblos y grupos. El estado, como tal, se refuerza en tanto que forma deseada de organización política a lo largo y ancho del planeta. Al mismo tiempo, algunos de los principios básicos de su propia naturaleza, como la no injerencia en los asuntos internos, empiezan a resquebrajarse. De ahí que las próximas décadas supongan un desafío importante, tanto para las potencias (responsables por el papel que desempeñan) como para las Naciones Unidas encargadas de establecer y aplicar el "código de conducta» en un mundo cada vez más desigual y más conflictivo. 
"Papers": Revista de Sociologia

\section{BIBLIOGRAFIA CITADA}

Obras de análisis:

Aron, R. (1962), Paix et guerre entre les nations, Paris, Calman Levy.

Bull, H. (1977), The Anarchical Society. A Study of Order in World Politics, Londres, Macmillan.

Colard, D. (1991), Les Relations Internationales de 1945 à nos jours, Paris, Masson (I². ed. 1977).

Hocking, B. y Smich, M. (1990), World Politics. An Introduction to International Relations, Nueva York, Harvester.

Kennedy, P. (1987), The rise and fall ot the Great Powers, Nueva York, Random House.

Morgenthau, H.). (1948), Politics among Nations. A struggle for power and peace, Nueva York, Alfred A. Kropf.

Nye, J. (1991), Bound to lead. The changing nature of American power, Nueva York, Basic Books, (1 ${ }^{2}$, ed. 1990).

Pfaff, W. (1990), "The Might, and Sense, of a Superpower", The International Herald Tribune, 14 diciembre.

Russett, B. y Starr, H. (1989), World Politics. The Menu for Choice, New York, Freeman and Company, (1 $1^{\text {a }}$. ed. 1981).

Waltz, K. (1979), Theory of International Politics, Reading, Addison-Wesley.

Obras de referencia:

Anuario El Pais 1993, Madrid, Editorial El País.

Informe sobre el Desarrollo Mundial 1992, Washington D.C., Banco Mundial.

Kidron, M. y Smith, D. (1991), The new state of war and peace. An international atlas, New York, Simon \& Schuster.

L'etat du monde. Edition 1993. Annuaire économique et géopolitique mondial París, Editions La Decouverte.

PNUD (1991), Desarrollo Humano. Informe 1991 (Resumen del Informe publicado por PNUD), Barcelona, Associació per a les Nacions Unides a Espanya.

PNUD (1992), Informe sobre desarrollo bumano 1992. Notas informativas ( $I$ ), Barcelona, Associació per a les Nacions Unides a Espanya.

SIPRI (1991), Amaments o desarmament? Compendi del SIPRI 1990, Bellaterra, Associació per a les Nacions Unides a Espanya/Centre d'Estudis sobre la Pau i el Desarmament.

SIPRI (1992), Sipri Yearbook 1992. World Armaments and Disarmament, Oxford, Sipri/Oxford University Press.

World Resources 1992-1993, New York, World Resources Institute/Oxford University Press. 